\title{
A new method to assess the deformations of internal organs of the abdomen during impact
}

P. Beillas* ${ }^{*}$ C. Helfenstein*, F. Rongieras * ${ }^{*}$, A. Le Ruyet*,

F. Berthet ${ }^{\circ}$, M. Tanter $\ddagger$ J-L. Gennisson $\ddagger$

* Univ. de Lyon; IFSTTAR, LBMC, Bron ; Univ. Lyon 1,

+ Hôpital Desgenettes, Lyon

- Lier-Transpolis, Lyon Saint Exupery

$\ddagger$ Institut Langevin, ESPCI ParisTech, Paris

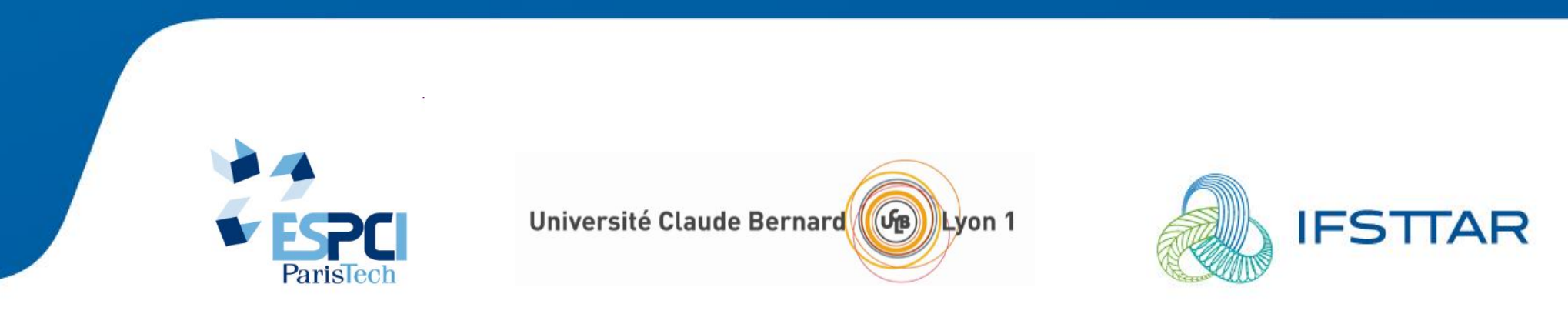




\section{Background and objectives}

- Context: automotive safety: Abdomen can be subjected to large \& rapid compressions

- Internal response difficult to observe (organ; region) $\rightarrow$ validation of modelling assumptions \& injury mechanisms?

- Recently: Howes et al. (Stapp, 2012)

- High speed bi plane Xray $\rightarrow$ 3D trajectories of implanted targets

- Objectives: Develop complementary protocols to observe the internal response of isolated organs or full abdomen

- Approach: ultrafast ultrasound imaging. = here: 5000 images per second - Aixplorer (Supersonic Imagine)

- Drawback: image quality reduced compared to classical B mode

- Two protocols presented: kidney + full body

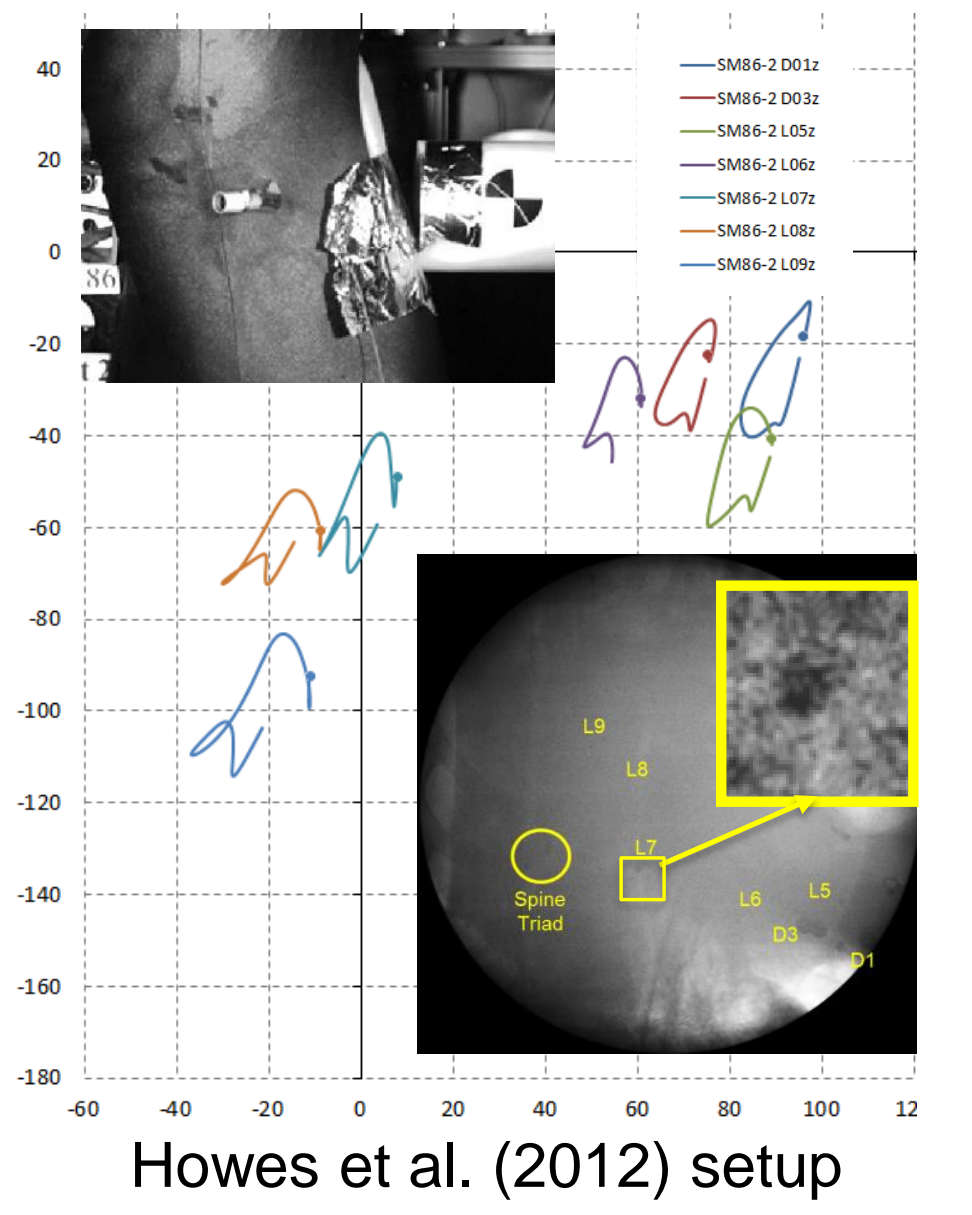




\section{Material and methods}

- Isolated organs: compressive testing of porcine or human kidneys

- Fresh organ, perfused at $80 \mathrm{mmHg}$ into artery

- Loading at 0.003 (non-destructive) and $0.3 \mathrm{~m} / \mathrm{s}$ (up to failure)

- Linear ultrasound probe $(0.2 \times 0.2 \mathrm{~mm}$, up to 5000 images $/ \mathbf{s})$
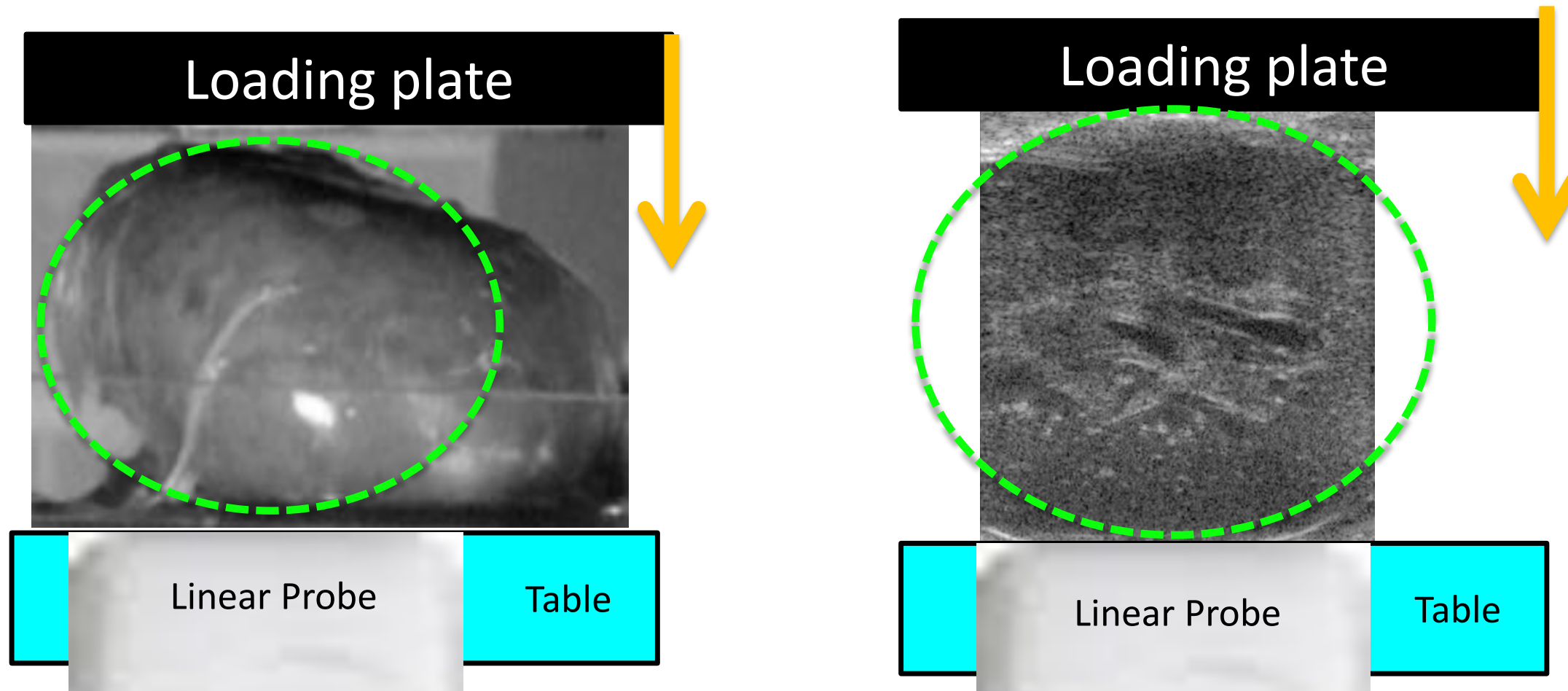


\section{Material and methods}

Full body impacts: 5 PMHS with test to test improvements. Typically:

- Preparation (Day 1 after thawing):

- Incision on linea alba

- Tracheotomy for lung inflation

- Catheter in iliac artery for perfusion

- Gas removed from intestines, antibiotics+saline injected

- Colon emptied and filled (gel)

Test setup:

- Inverted mount (Howes et al., 2012)

- Adjustable impact location, stroke \& speed (1 to $7 \mathrm{~m} / \mathrm{s}$ )

- Cylinder (tip: flat or sphere), belt

- Curved probe (192 elements) on positioning arm (subject) or inside impactor 2000 images/s

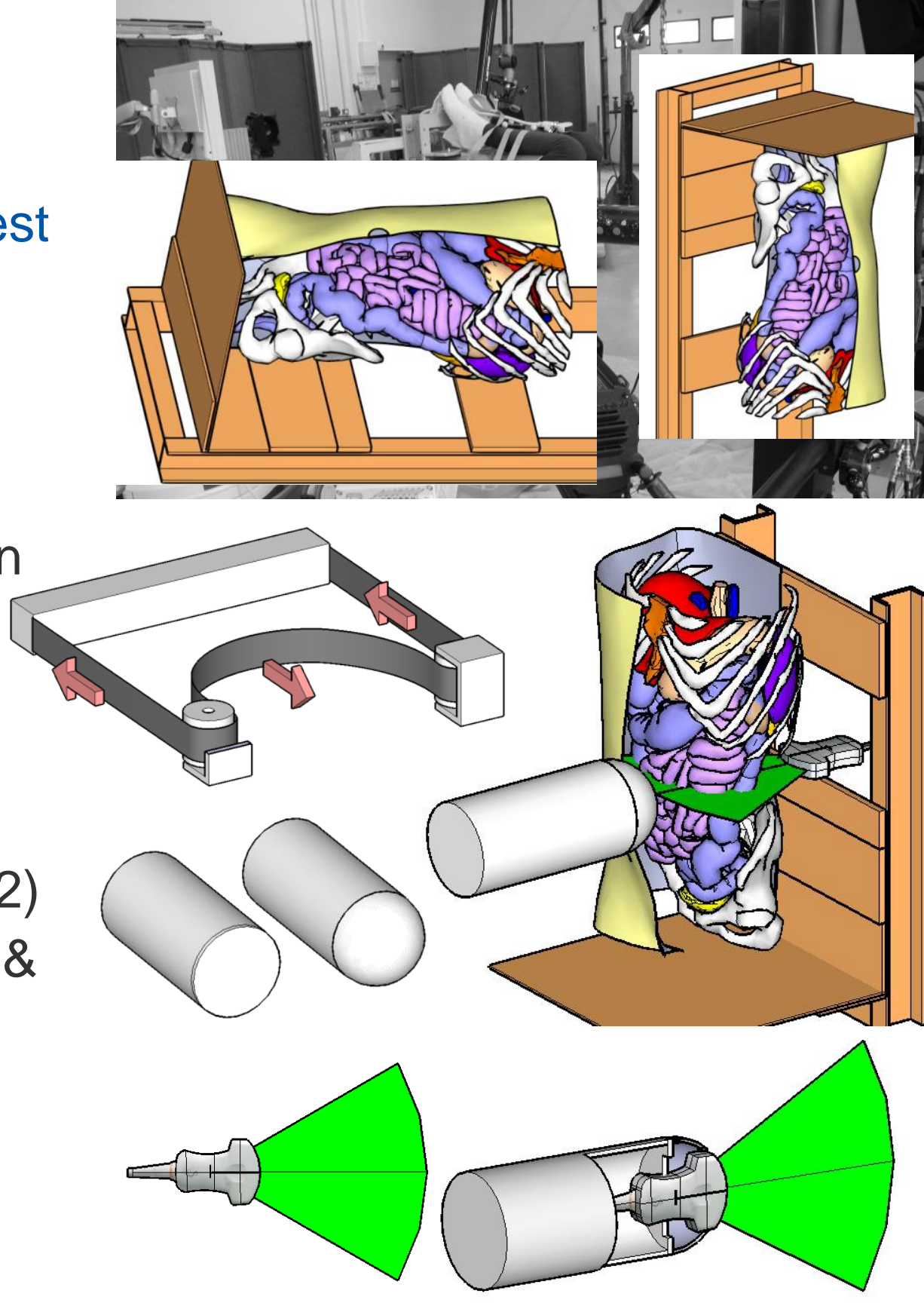




\section{Material and methods}

Full body impacts (cont'd)

Testing (day 2): lungs inflated prior to each test. 2 regions of interest :

1) descending colon axial plane; cylinder or belt, limited stroke, no perfusion

2) liver/diaphragm sagittal plane $\rightarrow$ Removal of 2 floating ribs;

a) cylinder or belt, limited stroke, no perfusion

b) Cylinder, large stroke, perfusion

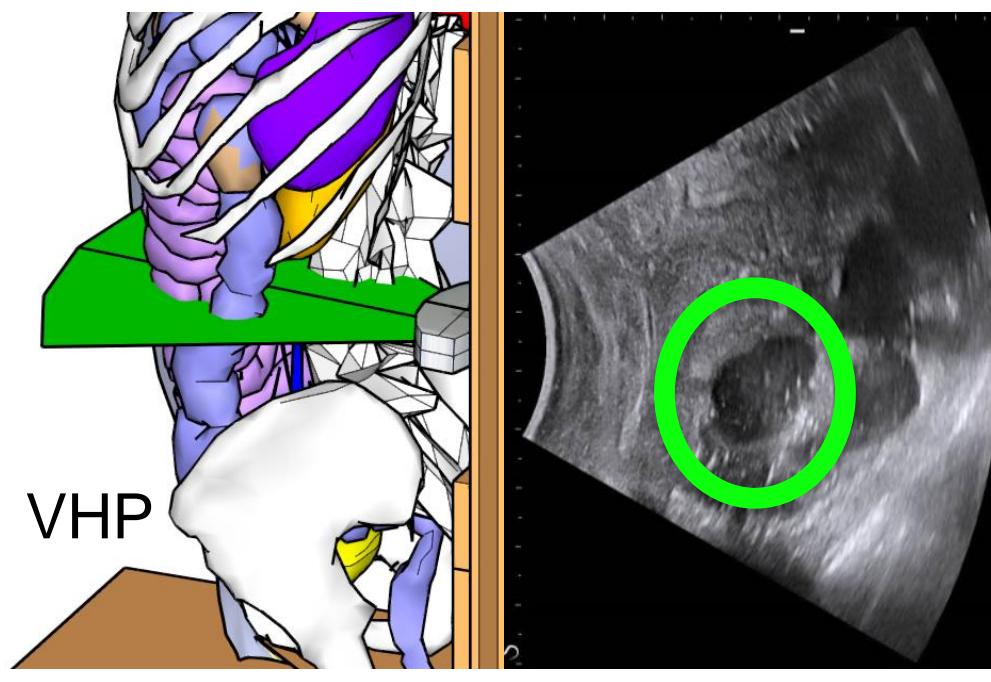

Total: up to 20 tests per subject... Instrumentation and geometry :

- Force, acceleration, cameras...

- CT-scan, surface scan, B-mode
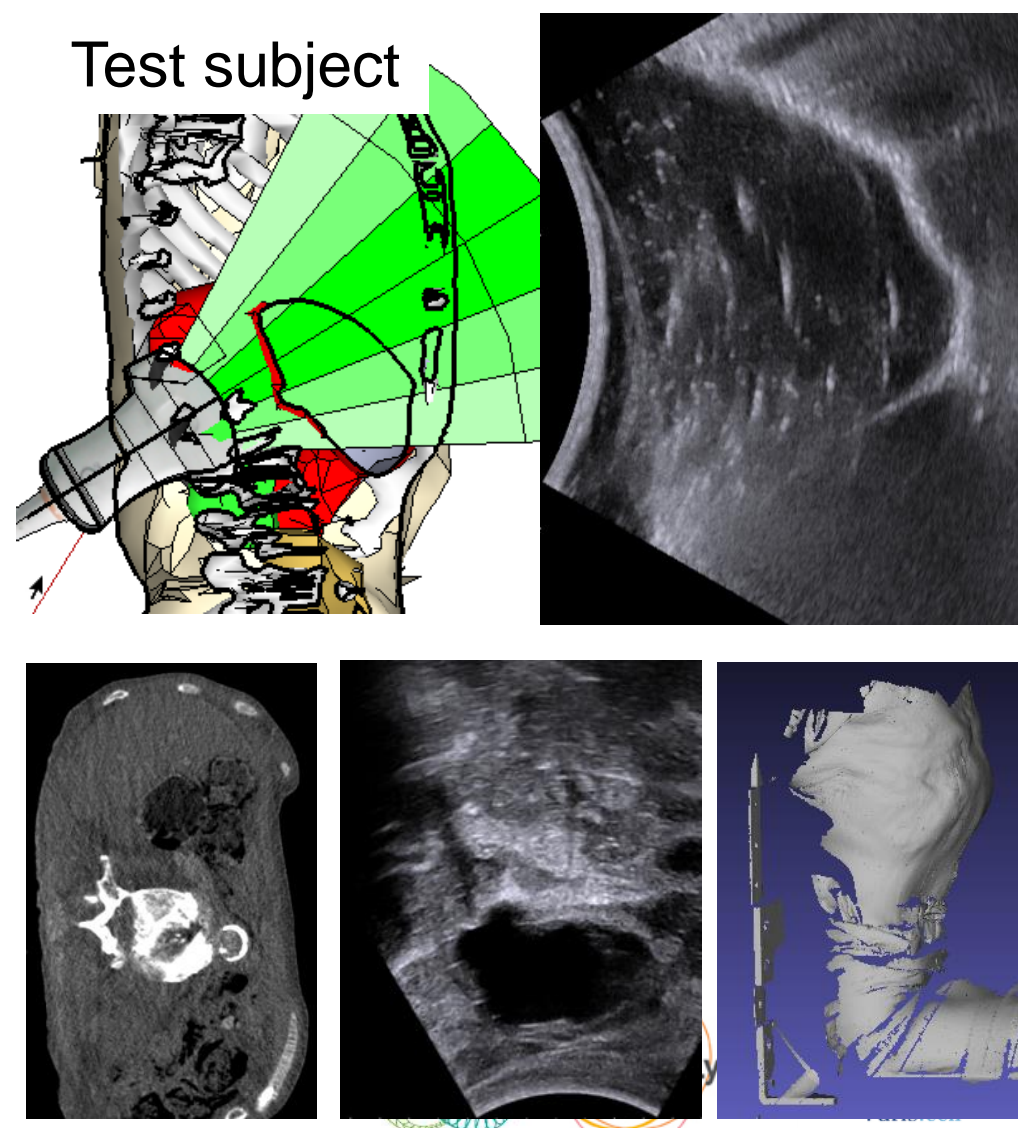


\section{Results: isolated kidney}

- Example of test up to failure

- sudden motion towards peak force $\rightarrow$ time of failure

Uniaxial strains by zone (target tracking) always possible

- E.g. effect of strain rate (paired tests on porcine organs)

Cortex

impactor

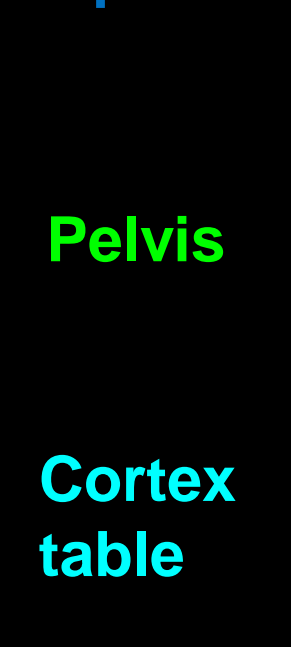

B
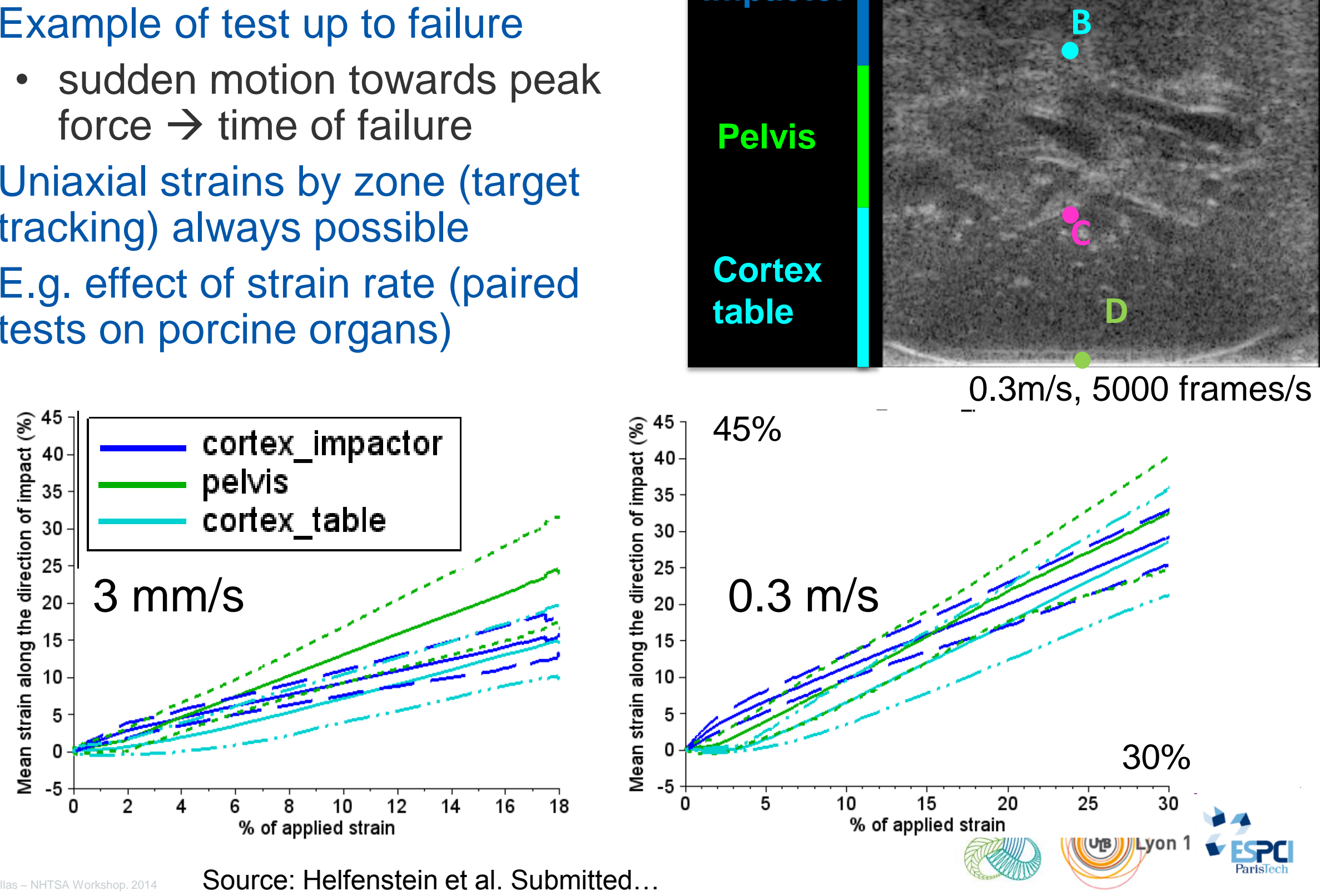

Source: Helfenstein et al. Submitted... 


\section{Results: isolated kidney: strain maps}

- Challenges: noise, possible through plane motion... (Typical 2D correlation not ok...)

- Approach developed: (1) automatic target tracking (Preibisch et al., 2010) (2) FE model based interp.

- Preliminary results:

- Predicts manual target tracked

- Strain map respecting boundary conditions...

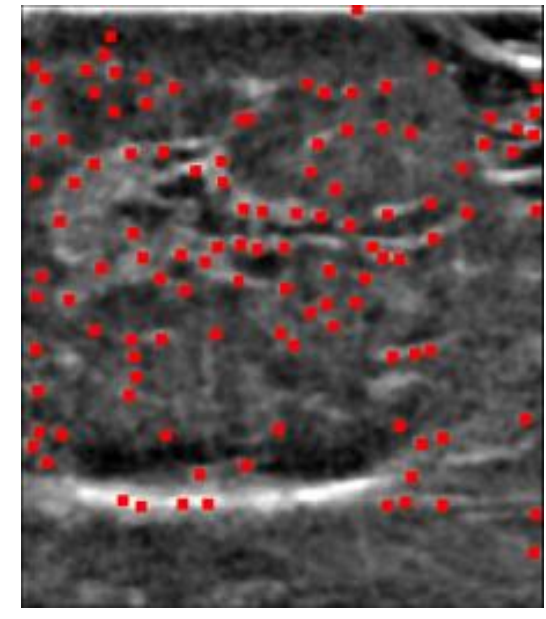

- Need more validation work...

\section{Strain map}

Preibisch et al. (2010). Nature Methods
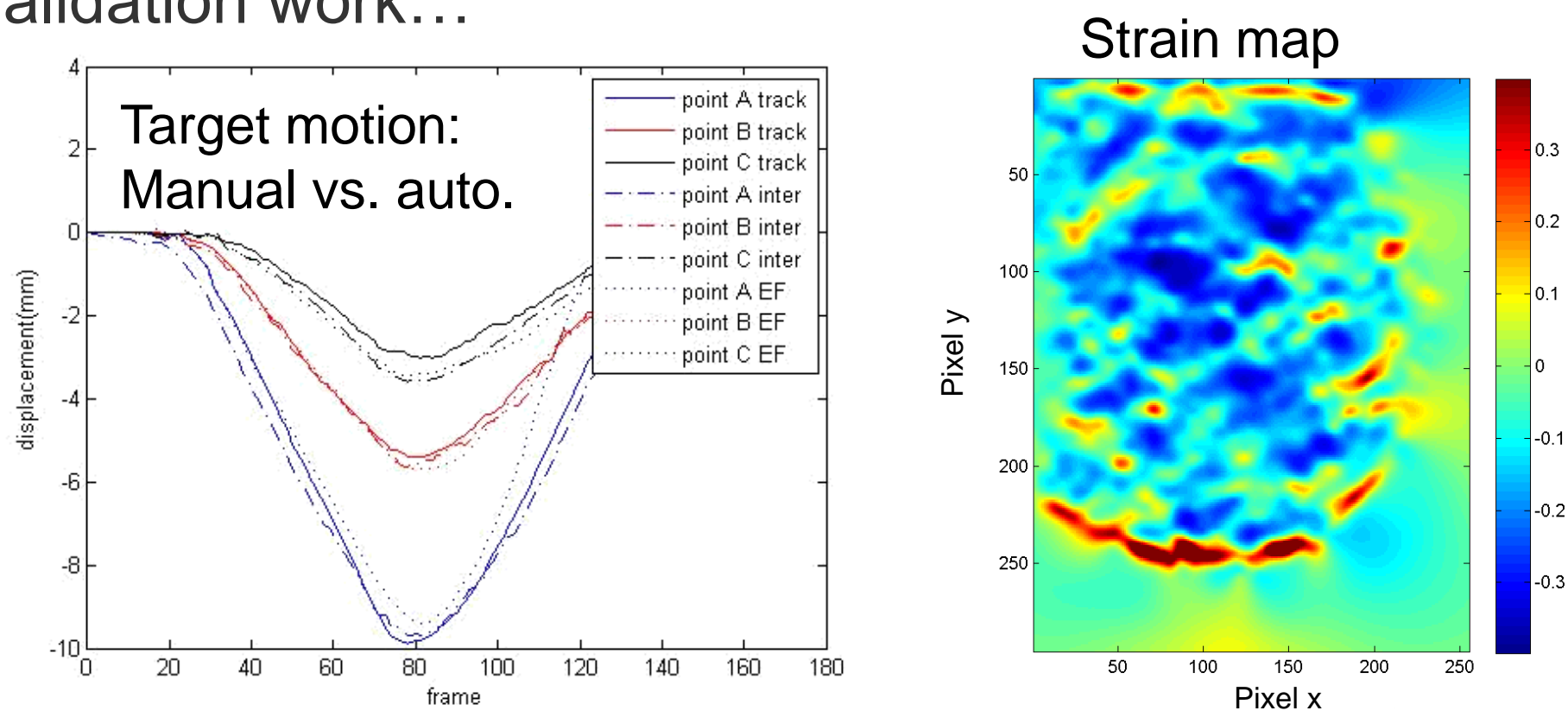
Results: Full body tests - liver (subject 3)

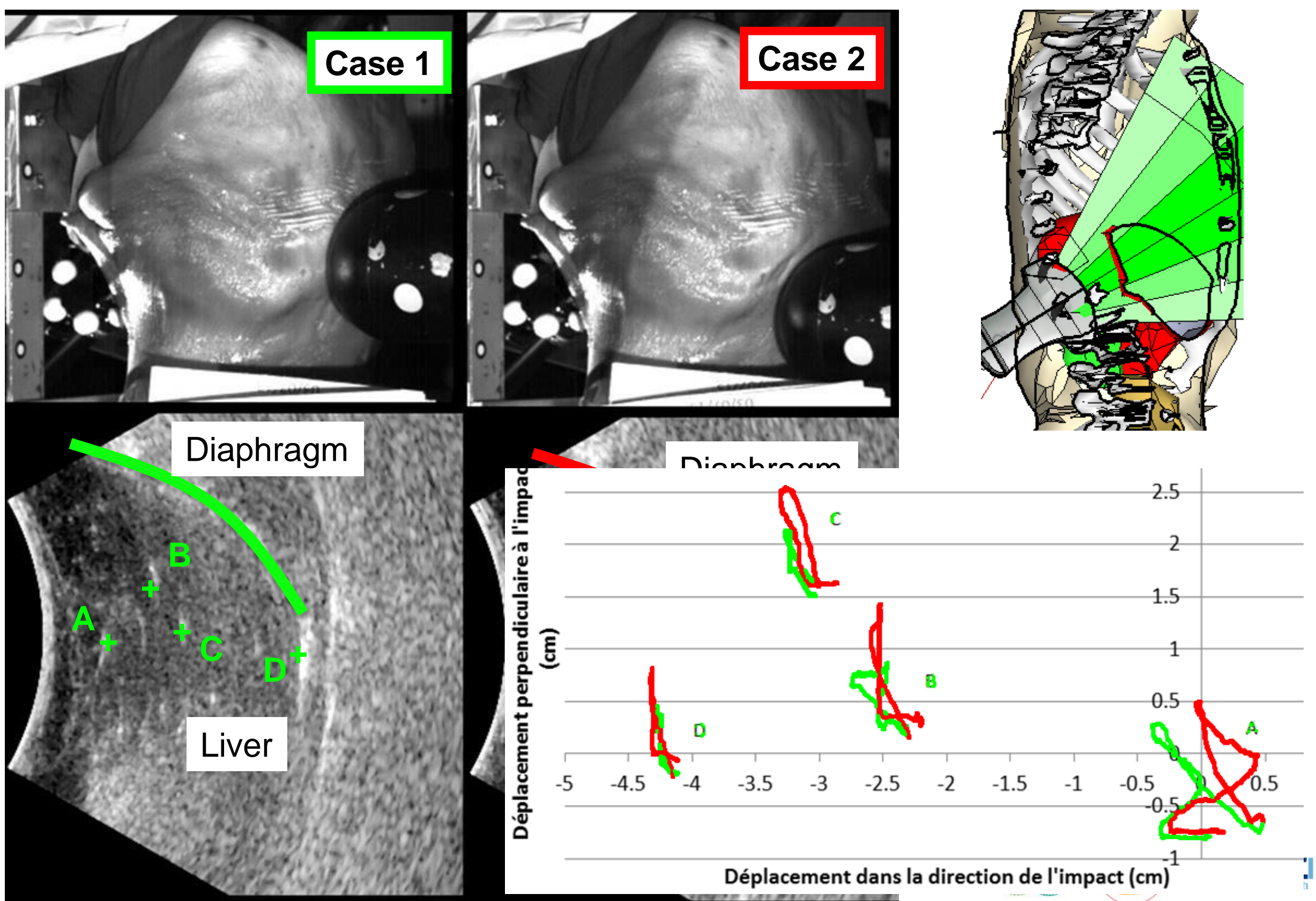




\section{Results: liver (subject 5)}

- Effect of velocity (movies frame rate adjusted for velocity difference)

$3 \mathrm{~m} / \mathrm{s}, 2000 \mathrm{im} / \mathrm{s}$

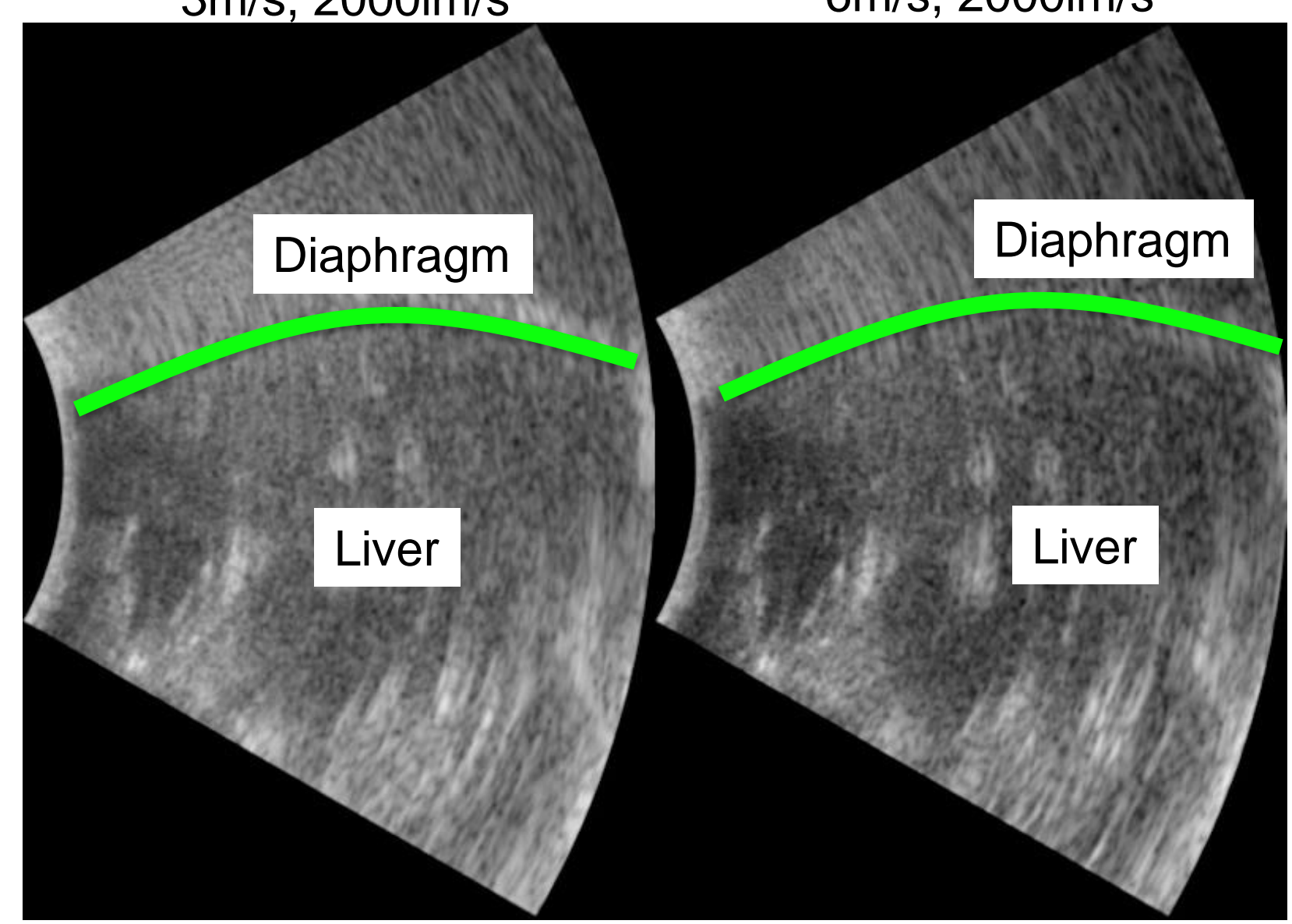

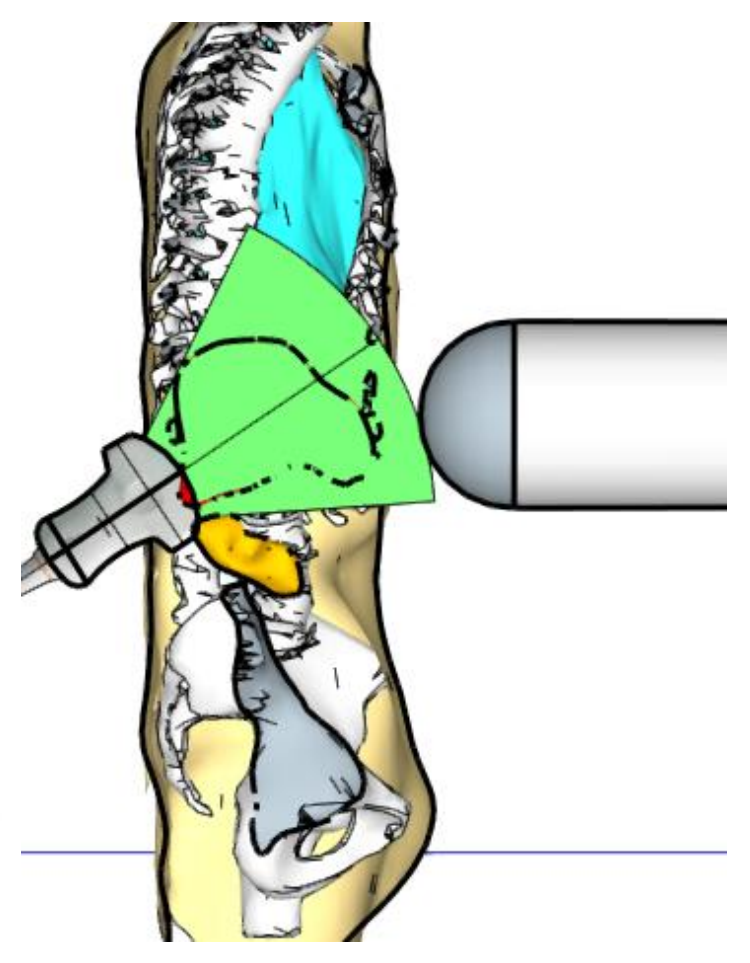

Approximate position 


\section{Results: dual probe}

- Colon and ... kidney (subject 2)

- Difficult to understand...

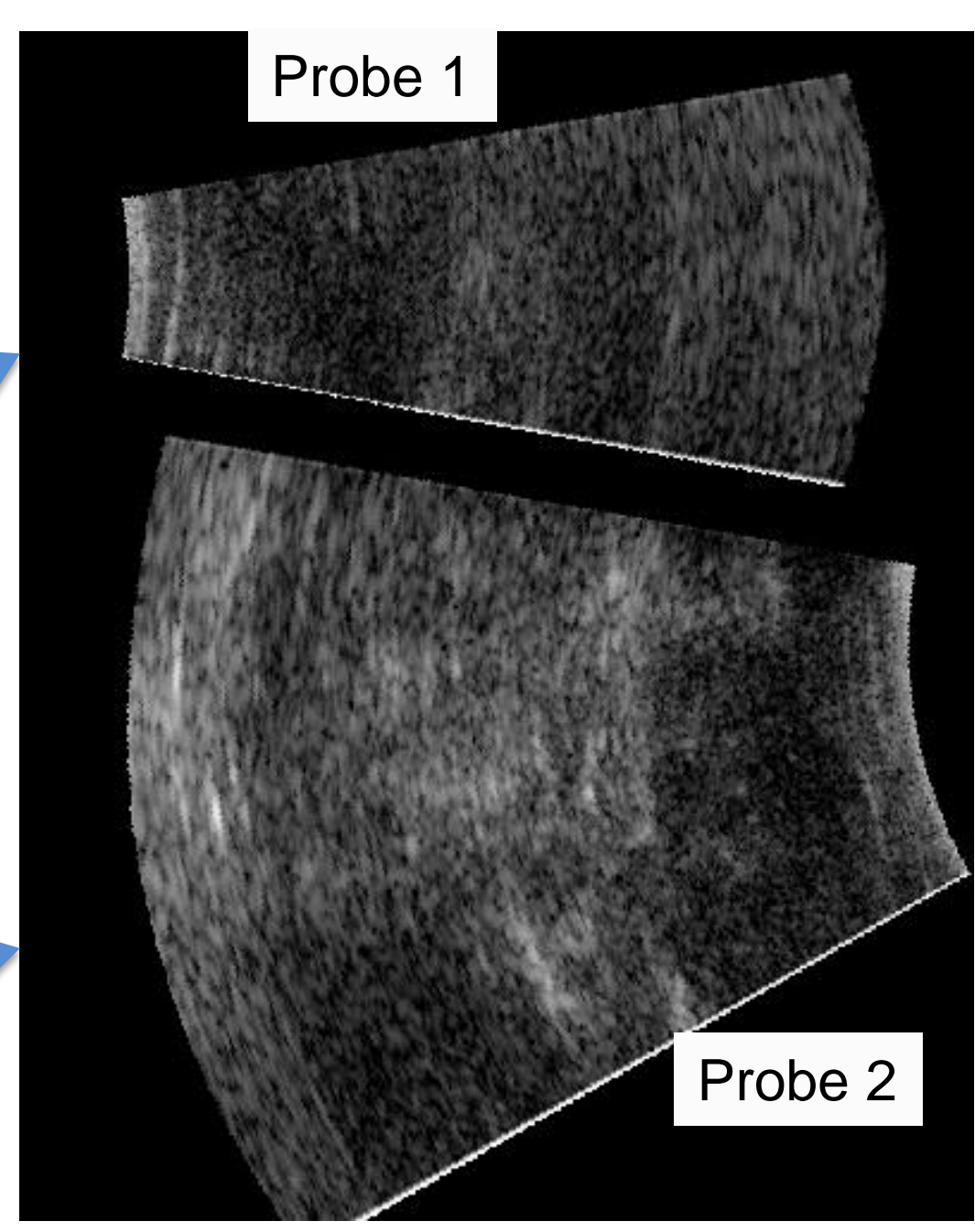

- Liver: results more promising (subject 4)...

2000 images per second 


\section{Discussion}

- New protocols developed/applied to isolated kidneys and 5 PMHS

- First quantifications, preliminary simulations

- Organ: non censored data? Whole body: mostly no abdo. injury

\section{Challenges and limitations}

- Numerous technical issues (trigger, software, ...) now solved

- Careful planning required (image quality/depth, through plane motion)

- PMHS model (not a live subject) and ultrasound requirements ...

- Gases: mostly solved (inverted body, gel) $\rightarrow$ response?

- Lung pressure helps control diaphragm position but risk of air leakage in abdomen, position of intestines?

- Perfusion issues whole body

- Floating rib removal for liver imaging

- Note: may have occurred in past testing without being noticed 


\section{Conclusions and Perspectives}

- Approach is promising...

- Seems flexible to study other PMHS configurations (e.g. isolated liver, side impact, thorax flexion) or volunteer

- Exploitation of available results: human FE model validation, strain maps processing

- Continue testing to increase sample numbers

+ some methodological improvements

- dual probe setup, 3D probe...

- Whole body: perfusion, non frozen PMHS 


\section{Acknowledgement}

- Financial support from:

- the Rhônes Alpes Region

- the GHBMC for the simulation work

- Co-authors and all personnel involved in the testing (Stéphane Ardizzone, Leila Ben Boubaker, Yves Caire)

- Supersonic Imagine... 